\title{
Nouvelle rubrique « controverse »
}

\section{Anneau gastrique}

\author{
J. Dargent - D. Nocca - J.-M. Chevallier \\ (C) Springer-Verlag France 2010
}

Nous inaugurons ici une rubrique « controverse ». Quel meilleur sujet que celui de l'opération qui a proprement marqué en France le coup d'envoi et le succès de la chirurgie de l'obésité, l'anneau modulable laparoscopique? D'une simplicité révolutionnaire et d'emblée jugé intéressant par la plupart des opérateurs, il a cependant vu le jour au milieu d'interrogations sur les principes mêmes de la chirurgie bariatrique. Les débats, loin de s'éteindre, rebondissent à présent sur les mérites propres d'une opération simple (trop simple ?), mais dont les contraintes sont souvent jugées trop importantes. Alors donc, l'anneau gastrique a-t-il véritablement un futur? Trois opérateurs répondent à leur façon : au lecteur de se faire une opinion...

Oui (J. Dargent) : l'anneau rend encore de grands services a priori dans toutes les situations d'obésité morbide ;

il a permis de comprendre et permettra d'utiliser mieux les mécanismes de la restriction alimentaire ; il restera une stratégie simple de recours à l'heure des techniques non invasives.

Non (D. Nocca) : on ne peut plus aujourd'hui concevoir une restriction rudimentaire parce que dysphagiante et obérée d'un matériel prothétique souvent défaillant ou dangereux ; la restriction moderne passe par une intervention qui rend de meilleurs services au plan métabolique et s'adapte mieux à la physiologie de l'obèse : la sleeve gastrectomie.

Oui, mais (J.-M. Chevallier) : il est convaincant à présent de restreindre l'usage de l'anneau à certaines indications et d'évoluer vers d'autres techniques lorsque la connaissance de l'histoire du patient permet d'anticiper un meilleur résultat.

J. Dargent $(\bowtie)$

Polyclinique de Rillieux,

F-69140 Rillieux-la-Pape, France

e-mail : jerome.dargent@polyclinique-rillieux.fr

D. Nocca $(\bowtie)$

Service de chirurgie viscérale,

hôpital Saint-Éloi,

80, avenue Augustin-Fliche,

F-34295 Montpellier, France

e-mail : d-nocca@chu-montpellier.fr

J.-M. Chevallier $(\bowtie)$

Secrétaire scientifique de la SOFFCO,

chirurgie digestive et de l'obésité,

hôpital européen Georges-Pompidou,

Assistance publique-Hôpitaux de Paris,

20, rue Leblanc, F-75908 Paris cedex 15, France

e-mail : jean-marc.chevallier@egp.aphp.fr 